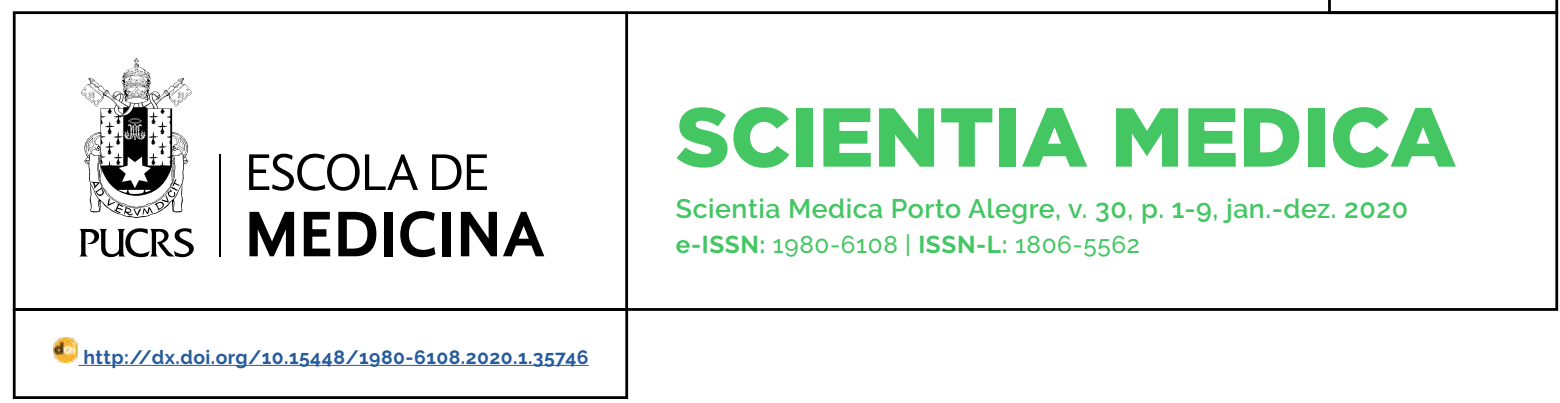

SEÇÃO: ARTIGO ORIGINAL

\title{
Características sociodemográficas e clínicas em nonagenários e centenários com e sem incontinência urinária
}

\author{
Sociodemographic and clinical characteristics in nonagenarians and centenarians with \\ and without urinary incontinence
}

\section{Vivian Ulrich ${ }^{1}$}

orcid.org/0000-0002-3893-711X

viviulrich@gmail.com

\section{Ângelo José Gonçalves \\ Bós ${ }^{2}$}

orcid.org/0000-0003-4901-3155 angelo.bos@pucrs.br

Recebido em: 18/9/2019

Aprovado em: $8 / 8 / 2020$

Publicado em: 03/11/2020.

\section{Resumo}

Objetivos: analisar as caracteristicas sociodemográficas e clínicas em individuos nonagenários e centenários com e sem incontinência urinária.

Métodos: trata-se de um estudo transversal e analítico, envolvendo 234 nonagenários e centenários avaliados nos seus domicilios, que foram aleatoriamente selecionados na cidade de Porto Alegre, RS. A presença de incontinência foi avaliada com relação às características clínicas e sociodemográficas cujas associações foram testadas pelo qui-quadrado e as diferenças nas médias testadas pelo t de Student usando o Programa Epi Info.

Resultados: a prevalência de incontinência urinária foi de $56,41 \%$, sendo $63.53 \%$ entre as mulheres e $37,5 \%$ entre os homens $(p=0,0003)$. A relação entre presença de perda de urina e de estado conjugal foi significativa $(p=0,0049)$, com maior frequência entre os viúvos. Os incontinentes saiam menos de casa, participavam menos de atividades físicas e sociais, tinham maior número de doenças crônicas e sintomas depressivos e pior pontuação na avaliação cognitiva. Em relação aos sintomas urinários, a maioria dos incontinentes referiu que a urina ou bexiga atrapalha a sua vida $(p<0,0001)$ e, que a urina ou bexiga, causa embaraço $(p<0,0001)$. Entre os participantes continentes, $25 \%$ relatou que a urina ou bexiga atrapalha a sua vida. Conclusões: a incontinência urinária foi mais frequente entre mulheres, participantes mais velhos, viúvos e individuos que moravam sozinhos. Apesar de maior prevalência entre as mulheres, chama atenção uma prevalência importante entre os homens. Ao fim, concluimos que a incontinência urinária impacta diretamente na vida social e na saúde de nonagenários e de centenários. Então, a incontinência urinária deve ser um importante componente na avaliação desse grupo. Observamos, também, que um número importante de nonagenários e de centenários apresentam incômodos relacionados à urina, mesmo sem incontinência urinária.

Palavras-chave: incontinência urinária, sintomas do trato urinário inferior, idoso de 90 anos ou mais, condições sociais, envelhecimento.

\section{Abstract}

Aims: to analyze the sociodemographic and clinical characteristics of nonagenarian and centenary individuals with and without urinary incontinence.

Methods: this is a cross-sectional and analytical study, involving 234 nonagenarians and centenarians evaluated in their homes, which were randomly selected in the city of Porto Alegre, RS. The presence of incontinence was assessed with respect to clinical and sociodemographic characteristics whose associations were tested by chi-square and the differences in means tested by Student's $t$ using the Epi Info program.

Results: the prevalence of urinary incontinence was $56.41 \%$, being $63.53 \%$ among women and $37.5 \%$ among men $(p=0.0003$. The relationship between the presence of urine loss and marital status was significant $(p=0.0049$ ), with greater frequency among widowers. Incontinents left the house less, participated less in physical and social activities, had a greater number of chronic diseases and depressive 
symptoms and a worse score in the cognitive assessment. Regarding urinary symptoms, most incontinents reported that urine or bladder interferes with their life $(p<0.0001)$ and that urine or bladder causes embarrassment ( $p<0.0001$ ). Among continent participants, $25 \%$ reported that urine or bladder disrupts their life.

Conclusions: urinary incontinence was more frequent among women, older participants, widowers and individuals who lived alone. Despite a higher prevalence among women, an important prevalence among men is noteworthy. We conclude that urinary incontinence directly impacts the social life and health of nonagenarians and centenarians. Therefore, urinary incontinence should be an important component in the evaluation of nonagenarians and centenarians. We also observed that a significant number of nonagenarians and centenarians present discomfort related to urine, even without urinary incontinence.

Keywords: urinary incontinence, lower urinary tract symptoms, aged 90 and over, social conditions, aging.

ABREVIATURAS: AMPAL, Projeto de Atenção Multiprofissional ao Longevo; APS, autopercepção de saúde; DCNT, doença crônica não transmissivel; GDS, escala de depressão geriátrica; HPB, hiperplasia prostática benigna; IBGE, Instituto Brasileiro de Geografia e Estatística; IU, incontinência urinária; MEEM, miniexame do estado mental.

\section{Introdução}

O aumento da longevidade da população é uma realidade na sociedade brasileira (1). A parcela com 80 anos ou mais é o grupo que mais cresce em todo o país, daí a importância de entender os fatores clínicos e sociais que envolvem esses indivíduos (2). Atualmente, no meio científico, se define o indivíduo com 80 anos ou mais como "idoso longevo". Esse grupo apresenta características morfológicas, fisiológicas, psicológicas e socioeconômicas diferentes de idosos mais jovens. A partir disso, surge a necessidade de estudos voltados para essa população $(3,4)$.

As condições de saúde da população idosa apresentaram melhoras na última década, mas há um aumento na prevalência de doenças crônicas (5). O conjunto das doenças crônicas comuns nos idosos é denominado "síndromes geriátricas" ou os gigantes da geriatria (6). Dentre as sindromes, a incontinência urinária (IU) é caracterizada por qualquer perda involuntária de urina (7), de prevalência relevante, que impacta na vida pessoal e social do idoso, além da sobrecarga dos cuidadores e do aumento dos custos para o sistema público de saúde (8).

Apesar do grande volume de estudos sobre o impacto da IU em idosos jovens, ainda são escassas as pesquisas que abordam a IU e suas características em idosos com mais de 90 anos. Assim, o objetivo desse estudo foi descrever as características sociodemográficas e clínicas em nonagenários e centenários da cidade de Porto Alegre, RS, Brasil. A intenção é proporcionar, desse modo, subsidios para a construção de políticas públicas de saúde particularmente na reabilitação da IU para essa população tão carente de um olhar especifico.

\section{Métodos}

Trata-se de um estudo transversal e analítico. A técnica de amostragem foi norteada pela seleção aleatória (sorteio) de setores censitários em todas as regiões administrativas (regiões do Orçamento Participativo) de Porto Alegre, capital do Rio Grande do Sul, sendo selecionados idosos de 90 anos ou mais que participaram da avaliação multidimensional do Projeto de Atenção Multiprofissional ao Longevo (AMPAL) da Pontificia Universidade Católica do Rio Grande do Sul (PUCRS). Os participantes deveriam ser domiciliados na cidade, sendo incluídos idosos com qualquer nivel funcional. O projeto é um estudo longitudinal aprovado pelo Comitê de Ética da PUCRS, pelo número de parecer 1.639.961. O projeto específico da análise dos dados de IU foi aprovado pelo mesmo comitê. pelo número de parecer 2.956.258. Em 2016, foram avaliados 245 nonagenários e centenários, mas somente 234 apresentaram respostas completas sobre as questões urinárias. A causa da perda amostral se deve à incapacidade de alguns participantes responderem a questões do instrumento de avaliação.

A presença de IU foi questionada através da pergunta "Você às vezes perde urina mesmo que pouca (uma gota)?", que poderia ser respondida tanto pelo participante quanto pelo familiar e/ou cuidador. Não foram utilizados outros instrumentos de rastreio para confirmação de diagnóstico, sendo assim, baseado no autorrelato 
dos participantes. Os sintomas urinários avaliados, para longevos com ou sem perda urinária, foram adaptados do instrumento Kings Health Questionnaire: o quanto a urina ou bexiga atrapalha a vida; se causa alguma vergonha ou constrangimento e se sente dificuldade ou desconforto ao urinar. Não foi avaliado tempo da IU nem houve confirmação clínica da IU.

$O$ instrumento multidimensional do AMPAL inclui, ainda, a avaliação das características sociodemográficas - sexo, estado conjugal, idade, cor, fato de saber ler e escrever e com quem reside -, clínicas e de estilo de vida - prática de atividade física, frequência com que sai de casa, recebe amigos, participa de atividades sociais, autopercepção de saúde geral (APS), número de doenças crônicas não transmissiveis, miniexame do estado mental (MEEM), escala de depressão geriátrica (GDS) e sintomas relacionados à urina. O estado conjugal foi agrupado em com companheiro (inclui casados), viúvo (sem companheiro) e outros (solteiro, separado ou divorciado). A idade foi categorizada em faixas etárias (90 a 94. 95 a 99 e 100 anos ou mais). Cor, agrupada em branca e não branca. Foram considerados fisicamente ativos os nonagenários e os centenários que responderam positivamente à questão "Nos últimos três meses praticou algum tipo de exercício físico ou esporte?", sendo considerado qualquer tipo de atividade física, aquela feita regularmente, pelo menos, uma vez por semana. O MEEM é um instrumento validado para uso com idosos. Foi traduzido para o português por Bertolucci et al. e possui questões que avaliam a linguagem, o cálculo, a atenção, a memória, a orientação em tempo e espaço e a evocação. A pontuação vai de zero a trinta e a classificação é realizada conforme a idade e a escolaridade (13). Os sintomas sugestivos de depressão foram avaliados pela GDS - 5, são cinco questões sobre esses sintomas, no qual o ponto de corte é 2 pontos (14).
Os dados foram analisados dividindo os participantes em com e sem IU. Participantes com IU foram considerados aqueles que relataram a presença de perda de urina mesmo em pouca quantidade. As frequências das características sociodemográficas e clínicas (variáveis categóricas) foram testadas pelo qui-quadrado. Já as diferenças nas médias da frequência de quem que sai de casa, recebe amigos e familiares e participa de atividades sociais, assim como do número de DCNT, desempenho no MEEM e número de sintomas depressivos no GDS-5. foram avaliados pelo teste $\mathrm{t}$ de Student. $\mathrm{O} \mathrm{p}<$ 0,05 foi considerado estatisticamente significativo. Niveis de significância entre 0,1 e 0,05 foram considerados indicativos de significância.

\section{Resultados}

A presente amostra totalizou 234 longevos, com média de idade 92,3 $\pm 3,62$ anos. A maioria dos idosos era do sexo feminino (72,65\%), viúvo (67,09\%), morava com familiar $(76,07 \%)$ e sabia ler e escrever (91,88\%).

A Tabela 1 descreve as características sociodemográficas de nonagenários e de centenários com e sem perda de urina. A prevalência de perda de urina total foi de $56,4 \%$ : nas mulheres, 63,5\% e, nos homens, 37,5\% ( $p<0,01)$. A relação entre presença de perda de urina e estado conjugal foi significativa $(p<0,01)$, com maior frequência entre os viúvos. $A$ idade dos participantes foi semelhante entre os grupos, apesar de a perda urinária ser mais frequente entre os centenários (71\%). A perda urinária prevaleceu entre os participantes de cor branca, sendo essa associação não significativa $(p=0,81)$. A média de anos de estudo foi menor nos incontinentes, que também apresentavam um percentual maior de analfabetos (0,39\%). Os participantes que referiram morar sozinhos, apresentaram maior frequência de perda de urina $(58,3 \%, p=0,82)$. 
TABELA 1 - Características sociodemográficas dos nonagenários e centenários com e sem perda de urina. Porto Alegre, 2016. $(n=234)$

\section{Perda urinária}

\begin{tabular}{|c|c|c|c|c|}
\hline & $\begin{array}{c}\text { Ausente } \\
(n=102)\end{array}$ & $\begin{array}{l}\text { Presente } \\
(n=132)\end{array}$ & $\begin{array}{c}\text { Total } \\
(n=234)\end{array}$ & $\mathbf{P}$ \\
\hline \multicolumn{5}{|l|}{ Sexo, n(\%) } \\
\hline Feminino & $62(36,5)$ & $108(63,5)$ & $170(72,7)$ & 0,0003 \\
\hline Masculino & $40(62,5)$ & $24(37,5)$ & $64(27,3)$ & \\
\hline \multicolumn{5}{|l|}{ Estado conjugal, n(\%) } \\
\hline Com companheiro (a) & $31(63.3)$ & $18(36,7)$ & $49(20,9)$ & 0,0049 \\
\hline Viúvo (a) & $58(36,9)$ & $99(63,1)$ & $157(67,1)$ & \\
\hline Outro' & $13(46,4)$ & $15(53,6)$ & $28(12,0)$ & \\
\hline \multicolumn{5}{|l|}{ Faixa etária, em anos, n(\%) } \\
\hline $90-94$ & $83(44,9)$ & $102(55,1)$ & $185(79,1)$ & 0,49 \\
\hline $95-99$ & $15(42,9)$ & $20(57,1)$ & $35(15,0)$ & \\
\hline $100^{+}$ & $4(28,6)$ & $10(71,4)$ & $14(6,0)$ & \\
\hline Cor branca, n(\%) & $83(43,2)$ & $109(56,8)$ & $192(82,0)$ & 0,82 \\
\hline Sabe ler e escrever, n(\%) & $96(44,7)$ & $119(55,4)$ & $215(91,9)$ & 0,38 \\
\hline \multicolumn{5}{|l|}{ Longevo mora com, n(\%) } \\
\hline Cuidador & $10(50,0)$ & $10(50,0)$ & $20(8,6)$ & 0,82 \\
\hline Familiar & $77(43,3)$ & $101(56,7)$ & $178(76,1)$ & \\
\hline Sozinho & $15(41,7)$ & $21(58,3)$ & $36(15,4)$ & \\
\hline
\end{tabular}

"outro estado conjugal se refere à situação de divorciado ou solteiro.

A Tabela 2 descreve as caracteristicas clínicas dos nonagenários e dos centenários com e sem perda de urina. Os nonagenários e os centenários incontinentes saem significativamente menos vezes por semana de casa $(p=0,02)$, fazem menos atividade fisica $(58,1 \%)$ e participam de menos atividades sociais, sendo esse indicativo de significância $(p=0,07)$. A autopercepção de saúde não apresentou relação significativa à perda urinária $(p=0,26)$. Apesar disso, os participantes com autopercepção de saúde regular relataram mais frequentemente perda urinária, que apresentou significativamente maior número de DCNT $(p=0,02)$ e menor pontuação do MEEM $(p=0,04)$. Os participantes com perda urinária apresentaram maior número de sintomas depressivos pelo GDS5 $(1,6 \pm 1,2)$, sendo essa diferença em relação aos sem perda urinária $(1,4 \pm 1,1)$ indicativa de significância estatística $(p=0,08)$.

As maiores frequências de perda de urina foram observadas nos participantes com sintomas relacionados à urina, sendo essa relação muito significativa para a urina ou a bexiga atrapalha a sua vida ( $p<0,0001$ ) e se a urina ou a bexiga causa embaraço ( $p<0,0001)$, e, indicativa de significância para desconforto ao urinar $(p=0,079)$. A frequência de participantes sem perda de urina foi de $12 \%$ entre os que referiram que a bexiga ou a urina atrapalha a vida "frequentemente ou muito" e $36 \%$ entre os referiram que a perda de urina atrapalha "às vezes ou mais ou menos" a sua vida (Tabela 2). A ausência de perda urinária foi de $29 \%$ entre o que referiram apresentar desconforto urinário. 
TABELA 2 - Caracteristicas clínicas dos nonagenários e centenários com e sem perda de urina. Porto Alegre, 2016. $(n=234)$

\section{Perda urinária}

\begin{tabular}{|c|c|c|c|c|}
\hline & $\begin{array}{c}\text { Ausente } \\
(n=102)\end{array}$ & $\begin{array}{c}\text { Ausente } \\
(n=132)\end{array}$ & $\begin{array}{c}\text { Total } \\
234(100)\end{array}$ & $\mathbf{P}$ \\
\hline Frequência que sai de casa & $12,3 \pm 11,7$ & $8,9 \pm 10,5$ & $10,36 \pm 11,1$ & 0,0207 \\
\hline \multicolumn{5}{|l|}{ Praticou atividade física, n(\%) } \\
\hline Não & $73(41,9)$ & $101(58,1)$ & $174(74,4)$ & 0,39 \\
\hline $\operatorname{Sim}$ & $29(48,3)$ & $31(51,7)$ & $60(25,6)$ & \\
\hline $\begin{array}{l}\text { Frequência que participa de atividades } \\
\text { sociais }\end{array}$ & $0,6 \pm 1,4$ & $0,3 \pm 0,9$ & $0,44 \pm 1,2$ & 0,0742 \\
\hline Frequência que recebe amigos familiares & $1,7 \pm 1,0$ & $1,8 \pm 0,8$ & $1,77 \pm 0,9$ & 0,63 \\
\hline \multicolumn{5}{|l|}{ Saúde geral, n(\%) } \\
\hline Má ou péssima & $9(45)$ & $11(55)$ & $20(8,66)$ & 0,25 \\
\hline Regular & $24(35,8)$ & $43(64,2)$ & $67(29,0)$ & \\
\hline Ótima ou boa & $69(47,9)$ & $75(52,1)$ & $144(62,3)$ & \\
\hline DCNT & $3,4 \pm 1,9$ & $3,9 \pm 2,2$ & $3,7 \pm 2,1$ & 0,0235 \\
\hline MEEM total & $21,6 \pm 5,9$ & $19,9 \pm 7,0$ & $20,6 \pm 6,6$ & 0,0420 \\
\hline GDS total' & $1,4 \pm 1,1$ & $1,6 \pm 1,2$ & $1,5 \pm 1,1$ & 0,0800 \\
\hline \multicolumn{5}{|l|}{ GDS depressão, n(\%) } \\
\hline Não & $86(45,5)$ & $103(54,5)$ & $189(80,8)$ & 0,22 \\
\hline $\operatorname{sim}$ & $16(35,6)$ & $29(64,4)$ & $45(19,2)$ & \\
\hline \multicolumn{5}{|l|}{ Urina atrapalha sua vida, n(\%) } \\
\hline Frequentemente ou muito & $6(11,8)$ & $45(88,3)$ & $51(21,8)$ & $<0,0001$ \\
\hline Às vezes ou mais ou menos & $20(36,4)$ & $35(63,6)$ & $55(23.5)$ & \\
\hline Nada & $76(59,4)$ & $52(40,6)$ & $128(54,7)$ & \\
\hline \multicolumn{5}{|l|}{ Urina causa embaraço, n(\%) } \\
\hline Não & $100(50,8)$ & $97(49,2)$ & $197(84,2)$ & $<0,0001$ \\
\hline Sim & $2(5,4)$ & $35(94,6)$ & $37(15,8)$ & \\
\hline \multicolumn{5}{|l|}{ Desconforto ao urinar, n(\%) } \\
\hline Não sente & $93(45,8)$ & $110(54,2)$ & $203(86,8)$ & 0,0793 \\
\hline Sente & $9(29,0)$ & $22(71,0)$ & $31(13,2)$ & \\
\hline
\end{tabular}

DCNT, doença crônica não transmissivel; MEEM, Miniexame do estado mental; GDS, Escala de Depressão Geriátrica. " média \pm desvio padrão. 


\section{Discussão}

No presente estudo participaram mais mulheres nonagenárias do que homens, corroborando com a projeção do IBGE para população do Brasil para o ano de 2016, que estima que $73 \%$ dos nonagenários são mulheres e $27 \%$ homens (2). Esses dados são também semelhantes ao observado na Pesquisa Nacional de Saúde, onde o percentual de mulheres com 90 anos ou mais era de $65.37 \%$, enquanto de homens na mesma faixa etária era $34,63 \%$ (15). Números esses que reforçam a tendência de termos mais mulheres longevas inseridas na sociedade.

Os autores tiveram muita dificuldade em comparar os resultados com a literatura, visto a ausência de publicações envolvendo as faixas etárias estudadas (nonagenários e centenários). A prevalência de IU é mais estudada em mulheres idosas e com resultados variados na literatura. Na pesquisa do Perfil dos Idosos do Rio Grande do Sul, 3.9\% dos entrevistados relataram perda urinária, enquanto no estudo de Marques et al., em Florianópolis, cujos participantes eram idosos e longevos, a prevalência foi de 29,4\% $(15,11)$. Apesar de a prevalência ser maior nas mulheres, o percentual de homens incontinentes observado na presente análise é maior que em outros estudos (10, 11). Essa baixa frequência de IU em homens nos outros estudos pode ser explicada pelo fato de a maioria dos trabalhos analisar idosos mais jovens. AIU nos homens pode estar relacionada à hiperplasia prostática benigna (HPB), ao tratamento do câncer de próstata, a incapacidades físicas, a sequelas de acidente vascular encefálico e a demências. A HPB pode ser encontrada em até 90\% dos homens acima de 90 anos $(16,17)$. Observamos poucos estudos dedicados à IU em homens idosos, menos ainda em nonagenários e centenários.

O presente estudo observou que, quanto maior a idade, maior foi a frequência de IU. Bolina et al. identificaram como fatores de risco para IU ter 70 anos ou mais e baixa escolaridade (18). O processo de envelhecimento pode induzir a mudanças no trato urinário, mas a continência também depende da capacidade de locomoção, habilidade manual, estado cognitivo e presença de doenças crônicas (19).

Em relação ao estado conjugal, $75 \%$ dos incontinentes eram viúvos, percentual maior que no estudo de Tavares et al., onde a frequência foi de $44.5 \%$. Os autores, que avaliaram pessoas mais jovens que no presente estudo, associam essa maior frequência à ausência de um companheiro - o que poderia ser um aliado no autocuidado do idoso (20). Dessa forma, nonagenários e centenários casados buscariam ajuda para a IU com mais frequência, justificando a menor prevalência nesse estado conjugal. Na presente pesquisa, a maior frequência de IU foi registrada entre os viúvos, tanto em homens quanto em mulheres.

A IU erroneamente está relacionada a uma condição natural do envelhecimento. A baixa escolaridade, por exemplo, pode influenciar na demora em procurar tratamento adequado (22). $\mathrm{Na}$ presente amostra, a frequência de incontinentes entre os que não sabem ler e escrever foi maior. A baixa escolaridade do individuo pode estar relacionada a uma maior situação de fragilidade e poderia influenciar na demora em procurar tratamento e esclarecimento da doença (21, 22). A literatura científica associa a relação entre escolaridade e cor ou etnia dos idosos brasileiros. Oliveira et al. observaram que idosos pretos e pardos predominavam na faixa de idade dos idosos jovens e de menor escolaridade, com piores indicadores sociais e de saúde (23).

A IU tem sido associada ao isolamento social (22). Entretanto, na presente amostra, os participantes com IU somente apresentaram menor frequência de sair de casa e realização das atividades sociais. Os nonagenários e os centenários entrevistados com IU receberam visita com frequência, semelhante aos continentes, sendo essa uma relação não descrita na literatura até a presente data. Dessa forma, a restrição social está condicionada à dificuldade de participar de atividades sociais e de sair de casa. Segundo Candido et al., a restrição social pode ocorrer devido ao medo do idoso perder urina em público, necessidade de ir ao banheiro muitas vezes e vergonha de estar com odor de urina (24). Mesmo 
adaptados ao uso de absorventes e/ou fraldas, persiste o desconforto, o estigma de ser uma situação associada à falta de higiene (22). Talvez esses motivos possam se aplicar aos participantes desse estudo, onde os incontinentes saíam de casa significativamente com menor frequência, além de praticarem menos atividade física e participarem de atividades sociais com uma frequência menor em relação aos continentes. Morsch et al. observaram que idosos com IU apresentaram 40\% menos chances de sair de casa. Os autores se referem ao possivel constrangimento causado pela IU como um fator limitante (25). No entanto, observamos que a IU não é um fator que interfere no fato do nonagenário e do centenário receber visitas, despertando o entendimento de que a restrição ao lar possa ter um motivo mais funcional que social.

Essa restrição para sair de casa pode desencadear outros problemas, como a depressão, e refletir na autopercepção de saúde (25). Apesar de não ser estatisticamente significativa, a maioria dos incontinentes apresentava APS ruim ou regular. Estudos em idosos mais jovens associam a presença de IU com mais sintomas depressivos, diminuição da função cognitiva e APS negativa (26). Os resultados da presente pesquisa demonstram que esses fatores são importantes também para nonagenários e centenários. A maior frequência de DCNT em participantes com IU observada, é apoiada por Bulut et al., que relataram a coexistência de sindromes geriátricas entre idosos, principalmente a IU, com 80 anos ou mais. Entre as sindromes geriátricas apontadas pelos autores, a depressão teve a prevalência reduzida entre os longevos (27). Mesmo assim, na presente análise, a IU foi positivamente relacionada à frequência maior de sintomas depressivos. Essa observação corrobora com a tese de que a IU afeta emocionalmente também nonagenários e centenários.

Observamos que a perda de urina gera incômodo para os incontinentes, atrapalhando a rotina ou causando algum tipo de constrangimento. Chama atenção o fato desses incômodos relacionados à perda urinária também estarem presentes nas pessoas que referiram à ausência de perda. No estudo realizado por Zanella, também se observou que mulheres continentes tinham queixas urinárias. Essas queixas podem estar relacionadas a uma perda urinária esporádica, não sendo suficiente para que elas se considerassem incontinentes (28). Silay et al. observaram que os participantes do estudo acreditavam que a IU fazia parte do envelhecimento e que não havia tratamento para ela, ocultando, muitas vezes, a condição (29). Elstad et al. entrevistaram homens e mulheres da comunidade e observaram que, entre os continentes, as queixas urinárias se referiam à urgência e à frequência que necessitavam ir ao banheiro. Dessa forma, a avaliação das queixas urinárias, como desconforto, embaraço e repercussão negativa sobre as atividades de vida diária causada pela urina, parece ser mais negligenciada que a avaliação da própria IU (30).

No presente estudo, observamos que a IU é uma condição frequente entre os nonagenários e os centenários. Apesar de maior prevalência entre as mulheres, chama atenção uma prevalência importante entre os homens. A incontinência urinária tem relação importante com o estado conjugal, a baixa frequência de sair de casa, a pouca participação em atividades físicas e sociais e com a saúde mental e física. Esse estudo teve como limitação a identificação da IU de forma autorreferida, podendo subestimar os resultados, principalmente, pelo fato que muitos individuos podem sentirse constrangidos em relatar a IU. Concluímos que a incontinência urinária impacta diretamente na vida social e na saúde de nonagenários e de centenários. E, que ela deve ser encarada como um importante componente na avaliação desse grupo. Por último, se faz importante destacar que, um número importante de nonagenários e de centenários apresentam incômodos relacionados à urina, mesmo sem incontinência urinária.

\section{Notas}

Este estudo é parte do resultado de dissertação de mestrado do Programa de Pós-Graduação em Gerontologia Biomédica da Pontifícia 
Universidade Católica do Rio Grande do Sul, de uma das autoras (VU), intitulada "Incontinência Urinária e Mortalidade em Nonagenários e Centenários do Projeto AMPAL".

\section{Apoio financeiro}

O presente trabalho foi realizado com apoio da Coordenação de Aperfeiçoamento de Pessoal Nivel Superior - Brasil (CAPES) - Código de Financiamento 001.

\section{Declaração de conflito de interesses}

Os autores declaram não haver conflitos de interesses relevantes ao conteúdo deste estudo.

\section{Contribuições dos autores}

Todos os autores fizeram contribuições substanciais para concepção, ou delineamento, ou aquisição, ou análise ou interpretação de dados; e redação do trabalho ou revisão crítica; e aprovação final da versão para publicação.

\section{Disponibilidade dos dados e responsabilidade pelos resultados}

Todos os autores declaram ter tido total acesso aos dados obtidos e assumem completa responsabilidade pela integridade destes resultados.

\section{Referências}

1. Pereira LF, Lenardt MH, Michel T, Carneiro NHK, Bento LF. Retrato do perfil de saúde-doença de idosos longevos usuários da atenção básica de saúde. Rev. enferm. UERJ. 2015;23(5):649-55. https://doi.org/10.12957/ reuerj.2015.5069

2. Instituto Brasileiro de Geografia e Estatística. Projeções da População do Brasil e Unidades da Federação por sexo e idade: 2010-2060 [Internet]. Rio de Janeiro: IBGE; 2018 [citado 30 mar 2019]. Disponivel em: https:// www.ibge.gov.br/estatisticas/sociais/populacao/ 9109-projecao-da-populacao $\cdot \mathrm{html}$ ? =\&t=resultados

3. Jorge MSG, Lima WG, Vieira PR, Vogelmann SC, Myra RS, Wibelinger LM. Caracterização do perfil sociodemográfico, das condições de saúde e das condições sociais de idosos octogenários. Saúde e Pesqui. 2017; 10(1):61-73. https://doi.org/10.17765/1983-1870.2017v10n1p61-73
4. Muniz EA, Aguiar MFS, Brito MCC, Freitas CASL, Moreira ACA, Araújo CRC. Desempenho nas atividades básicas da vida diária de idosos em Atenção Domiciliar na Estratégia Saúde da Familia. Rev. Kairós. 2016; 19(2):133-146.

5. Pimenta FB, Pinho L, Silveira MF, Botelho ACC. Fatores associados a doenças crônicas em idosos atendidos pela Estratégia Saúde da Família. Ciênc. Saúde Colet. 2015; 20(8):2489-2498. https://doi.org/10.1590/141381232015208.11742014

6. Vetrano DL, FoebelA, Marengoni A, Brandi V, Collamati A, Heckman GA, et al. Chronic diseases and geriatric syndromes: The different weight of comorbidity. Eur J Intern Med. 2016; 27: 62-67. https://doi.org/10.1016/j.ejim.2015.10.025

7. ICS. International Continence Society. Current definitions [Internet]. Bristol: ICS, 2020 [citado 14 abr. 2019]. Disponivel em: https://www.ics.org/terminology/113.

8. Ribeiro PCC, Banhato EFC, Guedes DV. Perfil clinico e uso de serviços de saúde em idosos. Revista HUPE, Rio de Janeiro, 2018;17(2):25-34. https://doi.org/10.12957/ rhupe.2018.40808

9. Benício CDAV, Luz MHBA, Lopes MHBM, Carvalho NAR. Incontinência Urinária: Prevalência e Fatores de Risco em Mulheres em uma Unidade Básica de Saúde. ESTIMA. 2016;14(4):161-168. https://doi.org/10.5327/ Z1806-3144201600040002

10. Giraldo-Rodríguez L, Agudelo-Botero M, Mino-León D, Álvarez-Cisneros T. Epidemiology, progression, and predictive factors of urinary incontinence in older community-dwellingMexican adults: Longitudinal data from the MexicanHealth and Aging Study. Neurourol Urodyn. 2019:38:1932-43. https://doi.org/10.1002/nau.24096

11. Marques LP, Schneider IJC, GiehL MWC, Antes $D L$, d'Orsi E. Demographic, health conditions, and lifestyle factors associated with urinary incontinence in elderly from Florianópolis, Santa Catarina, Brazil. Rev Bras Epidemiol. 2015;18(3):595-606. https://doi. org/10.1590/1980-5497201500030006

12. Instituto Brasileiro de Geografia e Estatística. Pesquisa Nacional de Saúde 2013: percepção do estado de saúde, estilos de vida e doenças crônicas - Brasil, Grandes Regiões e Unidades da Federação [Internet]. Rio de Janeiro: Instituto Brasileiro de Geografia e Estatística; 2014 [citado 15 abr 2019]. Disponivel em: ftp:// ftp.ibge.gov.br/PNS/2013/pns2013.pdf

13. Bertolucci PHF, Brucki SMD, Campacci SR, Juliano Y. O miniexame do estado mental em uma população geral: impacto da escolaridade. Arq Neuro-Psiquiatr. 1994; 52(1):1-7. https://doi.org/10.1590/S0004282X1994000100001

14. Almeida MSC. Efetividade da escala de depressão geriátrica de cinco itens em população idosa da comunidade. 133f. 2010. Tese (Doutorado) - Pontificia Universidade Católica do Rio Grande do Sul. Faculdade de Medicina. Programa de Pós-Graduação em Medicina e Ciências da Saúde. Porto Alegre: PUCRS, 2010. 
15. Bós DSG, Vianna MSS, Zanella AK. Prevalência de Incontinência Urinária em idosos gaúchos. In: BÓS ÂJG, Mirandola AR, Lewandowski A, Schirmer CL, organizadores. Perfil dos Idosos do Rio Grande do Sul. Porto Alegre: ESP/RS; 2015. p. 190-96.

16. Searcy JAR. Geriatric Urinary Incontinence.Nurs Clin N Am. 2017:52(3):447-455. https://doi.org/10.1016/i. cnur.2017.04.002

17. Colla C, Ghisleni AP, Paiva LL. Perfil de usuários que buscam atendimento fisioterapêutico para incontinência urinária em um centro de saúde do municipio de Porto Alegre. Rev. Saúde Públ. 2015;8(3):45-54.

18. Bolina AF, Dias FA, Santos NMF, Tavares DMS. Incontinência urinária autorreferida em idosos e seus fatores associados. Rev Rene. 2013;14(2):354-63.

19. Suhr R, Lahmann NA. Urinary incontinence in home care: a representative multicenter study on prevalence, severity, impact on quality of life, and risk factors. Aging Clin Exp Res. 2018;30(6):589-594. https://doi. org/10.1007/s40520-017-0816-6

20. Tavares DMS, Bolina AF, Dias FA, Santos NMF. Qualidade de vida de idosos com incontinência urinária. Rev Eletr Enf. 2011;13(4):695-702. https://doi.org/10.5216/ree.v13i4.12488

21. Lenardt MH, Carneiro NHK, Binotto MA, Setoguchi LS, Cechinel C. Relação entre fragilidade física e características sociodemográficas e clínicas de idosos. Esc Anna Nery. 2015;19(4):585-592. https://doi. org/10.5935/1414-8145.20150078

22. Matos MAB, Barbosa BLA, Costa MC, Rocha FCV, Almeida CAPL, Amorim FCM. As repercussões causadas pela incontinência urinária na qualidade de vida do idoso. Rev Fund Care Online. 2019;11(3):567-575. https://doi.org/10.9789/2175-5361.2019.v11i3.567-575

23. Oliveira BLCA, Thomaz EBAF, Silva RA. Associação da cor/raça aos indicadores de saúde para idosos no Brasil: um estudo baseado na Pesquisa Nacional por Amostra de Domicilios (2008). Cad Saúde Pública. 2014:30(7):1-15

24. Candido FJLF, Matnei T, Galvão LC, Santos VLJ, Santos MC, Sarris AB, et al. Incontinência urinária em mulheres: breve revisão de fisiopatologia, avaliação e tratamento. Visão acadêmica. 2017;18(3):67-80. https:// doi.org/10.5380/acd.v18i3.54506

25. Morsch P, Pereira GN, Navarro JHN, Trevisan MD, Lopes DGC, Bós ÂJG. Características clínicas e sociais determinantes para o idoso sair de casa. Cad Saúde Pública. 2015, 31(5):1025-1034. https://doi.org/10.1590/ 01021-311X00053014

26. Kessler M, Facchini LA, Soares UM, Nunes BP, França $\mathrm{SM}$, Thumé E. Prevalência de incontinência urinária em idosos e relação com indicadores de saúde física e mental. Rev. Bras. Geriatr. Gerontol. 2018;21(4):409419. https://doi.org/10.1590/1981-22562018021.180015
27. Bulut EA, Soysal P, Isik AT. Frequency and coincidence of geriatric syndromes according to age groups: single-center experience in Turkey between 2013 and 2017. Clin Interv Aging. 2018; 4(13):1899-1905. https:// doi.org/10.2147/ClA.S180281

28. Zanella AK. Avaliação da consciência da musculatura do assoalho pélvico e sua relação com a incontinência urinária em idosas [tese]. Porto Alegre (RS): Pontifícia Universidade Católica do Rio Grande do Sul; 2016.

29. Silay K, Akinci S, Ulas A, Yalcin A, Silay YS, Akinci $M B$, et al. Occult urinary incontinence in elderly women and its association with geriatric condition. Eur Rev Med Pharmacol Sci. 2016;20(3):447-51.

30. Elstad EA, Taubenberger SP, Botelho EM, Tennstedt $\mathrm{SL}$. Beyond incontinence: The stigma of other urinary symptoms. J Adv Nurs. 2010;66(11):2460-2470. https:// doi.org/10.1111/j.1365-2648.2010.05422.x

\section{Vivian Ulrich}

Mestra em Gerontologia Biomédica pela Pontifícia Universidade Católica do Rio Grande do Sul (PUCRS), em Porto Alegre, RS, Brasil.

\section{Ângelo José Gonçalves Bós}

Doutor pela Tokai University, Japão. Professor titular da Escola de Medicina da Pontificia Universidade Católica do Rio Grande do Sul (PUCRS), em Porto Alegre, RS, Brasil.

\section{Endereço para correspondência}

Vivian Ulrich

Pontificia Universidade Católica do Rio Grande do Sul Av. Ipiranga, 6681, prédio 40, sala 806

Partenon, 90619900

Porto Alegre, RS, Brasil 